\title{
A Study on Teleoperation of a Mobile Robot Using Haptic Feedback Yun Bae Kim ${ }^{1, a}$, Gi Sang Choi ${ }^{1, b}$ \\ ${ }^{1}$ Dept. of Electrical \& Computer Engineering, Univ. of Seoul, Siripdae-gil 163, Dongdaemun-gu, Seoul, Korea
}

arladbsqo321@gmail.com, bsimpson@uos.ac.kr

\section{Keywords: Haptics, Teleoperation, Mobile robot, Obstacle avoidance}

\begin{abstract}
Haptic feedback force is often suggested to complement visual information through the sense of touch to improve efficiency and safety in the teleoperation of mobile robot. The efficiency and safety of teleoperation are strongly dependent upon how haptic feedback is presented to the operator. In this study a haptic feedback scheme for teleoperation of mobile robot is developed and its effectiveness is experimentally verified. Experimental results show that the developed scheme improves the quality of mobile robot teleoperation in terms of improvement in efficiency and safety.
\end{abstract}

\section{Introduction}

Recently, teleoperation is being widely adapted for much hazardous work in factory environment. Well known examples of robot based teleoperation are inspection of underwater structures[1] and cleaning of nuclear plants[2]. In [3] Haptic teleoperation for construction robot based on virtual reality was developed. In teleoperation, a simple way of presenting telepresence is to show the environmental data to user visually. But in many applicatons visual data is insufficient because of limited visual fields of cameras. For such cases teleoperation with haptic feedback can be effective. There have been several researches on haptic interfaces for mobile teleoperation. Concept of virtual cone for intuitive mobile teleoperation with haptic feedback was proposed in [4]. In [8], Effectiveness of haptic force feedback is tested and analyzed for three different control methods. Variable feedback gain method depending on approaching speed to obstacle is developed in [7].

In this paper exponential haptic force feedback is proposed. In other research force feedback is linearly proportional to the distance to obstacles[5-7]. However, presence of obstacle can hardly be recognized with linear increase of haptic force in many applications. In this study the exponential relationship between the distance to the obstacle and the haptic force is adpated in order to provide substantial feeling of presence of obstacle on the way of robot motion. Expeimental verification showed that the proposed scheme improves the performance of teleoperation of mobile robot.

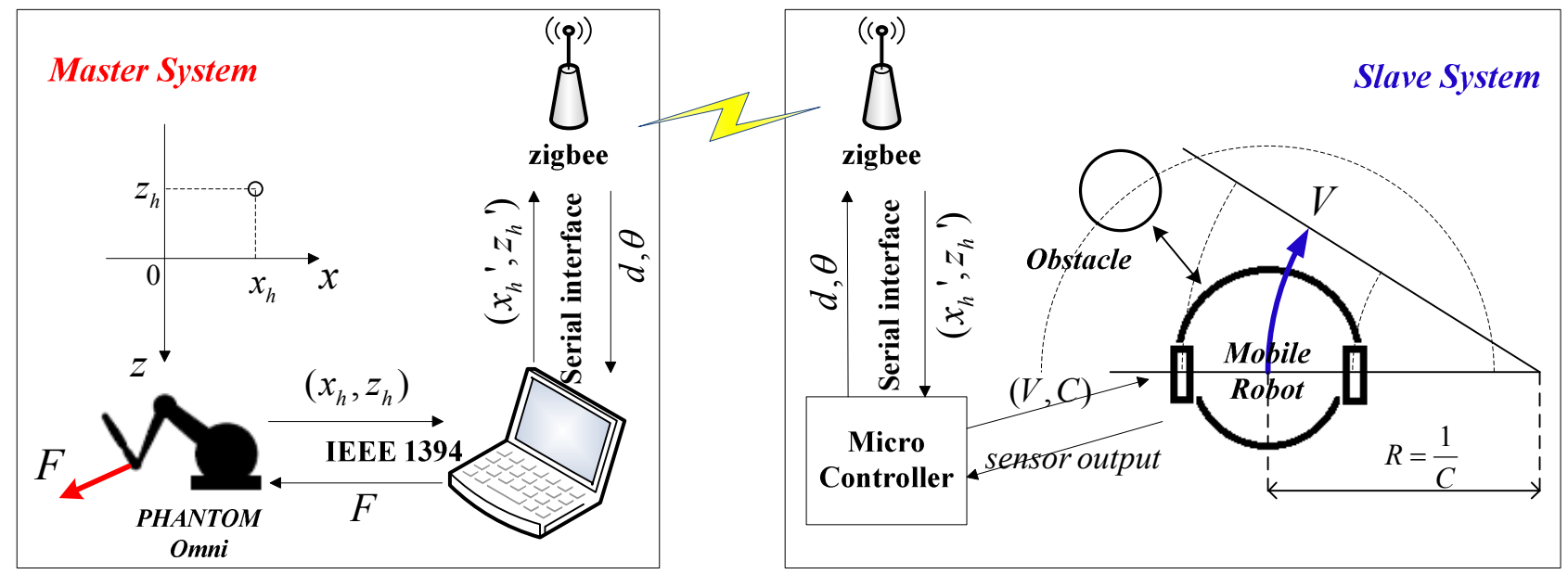

Fig. 1 Configurations of master device and mobile robot(Overall System) 


\section{Overview of the Haptic Teleoperation System}

Haptic teleoperation system uses haptic operating device that generates haptic forces as feedback information. The haptic teleoperation system considered in this study is schematically illustrated in Fig. 1. PHANTOM Omni haptic input device from SensAble Corp. is adapted for the system. Human operator gives motion commands to the computer using the haptic device connected to the computer with IEEE1394 port. Then the 2-dimensional position information $\left(x_{h}, z_{h}\right)$ from the haptic device is send to the mobile robot controller using the Zigbee radio frequency communication. Then, in the microcontroller of the mobile robot the position data from the haptic device is converted to linear velocity $(V)$ and curvature of mobile robot $(C)$. During the operation obstacles are detected by ultrasonic sensors installed on the robot, and its distance $(d)$ and orientation $(\theta)$ are measured and the corresponding haptic feedback force $(F)$ to prevent the collision is calculated, and sent back to the master computer to give haptic feedback force back to user. This bilateral haptic control system is expected to prevent collision with obstacles and consequently more effective and safer teleoperation.

\section{Command Strategy}

Position-speed command strategy is widely used for remote control applications of mobile robots. This method is intuitive for user and it is useful for feedback force rendering as described in [5]. Local position of the haptic device point is mapped to linear velocity and curvature of mobile robot as follows:

$$
\begin{aligned}
& \left(\begin{array}{l}
V \\
C
\end{array}\right)=\left(\begin{array}{cc}
k_{V} & 0 \\
0 & k_{R}
\end{array}\right)\left(\begin{array}{l}
q_{z} \\
q_{x}
\end{array}\right) \\
& q_{z}=\left\{\begin{array}{cc}
-z_{h}, & \left|z_{h}\right|>z_{d z} \\
0, & \left|z_{h}\right| \leq z_{d z}
\end{array}\right. \\
& q_{x}=\left\{\begin{array}{cc}
x_{h}, & \left|x_{h}\right|>x_{d z} \\
0, & \left|x_{h}\right| \leq x_{d z}
\end{array}\right.
\end{aligned}
$$

Dead zone of the pointing device is reflected in Eq. 2 and Eq.3. The dead zone prevents unintended movements of the robot due to unintended minute motion or tremor of user. In mobile robot controller, conversion of the curvature and the linear velocity to velocities of left and right wheels is performed based on Eq. 4-6. In these equaions, $V_{l}$ and $V_{r}$ are velocities of left and right wheels, respectively.



Fig. 2 Kinematics of mobile robot that has 2 wheels used in this study

$$
a=\frac{W V}{2 R}=\frac{W V C}{2} \text { where }\left\{\begin{array}{c}
(V+a) \cdot t=(R+W / 2) \cdot \theta \\
(V-a) \cdot t=(R-W / 2) \cdot \theta \\
V \cdot t=R \cdot \theta
\end{array}\right.
$$




$$
\begin{aligned}
& V_{l}=V+a=V+\frac{W V C}{2} \\
& V_{r}=V-a=V-\frac{W V C}{2}
\end{aligned}
$$

\section{Haptic Feedback Algorithm}

In the proposed haptic teleoperation system, feedback forces are generated to recover the neural position of the haptic device (recovery force, $F_{i, x}, F_{i, z}$ ), and also to give notion of approaching obstacles to the human operator (environmental force $F_{e, x}, F_{e, z}$ ). Thus the force applied to user by the haptic device is computed as a function of environmental and recovery forces as:

$$
F=f\left(F_{e, x}, F_{e, z}, F_{i, x}, F_{i, z}\right)
$$

Recovery force is a force required to move back to the original neutral position of the haptic device. The magnitude of the recovery force is designed to be a constant $k_{i}$. Including the dead zone, the formula for the recovery force becomes:

$$
\begin{aligned}
& F_{i, x}=\left\{\begin{array}{cc}
-k_{i} \cdot \operatorname{sgn}\left(x_{h}\right), & \text { if }\left|x_{h}\right|>x_{d z} \\
0 & \text { if }\left|x_{h}\right| \leq x_{d z}
\end{array}\right. \\
& F_{i, z}=\left\{\begin{array}{cc}
-k_{i} \cdot \operatorname{sgn}\left(z_{h}\right), & \text { if }\left|z_{h}\right|>z_{d z} \\
0 & \text { if }\left|z_{h}\right| \leq z_{d z}
\end{array}\right.
\end{aligned}
$$

There have been numerous research efforts on teleoperation of mobile robot using haptic feedback [5-8]. In these studies various force feedback algorithms have been tried. In [5,6], feedback force depends only on the distance between obstacles and robot, but in $[7,8]$ the force is dependent not only upon the distance but also upon the position of control device. Recovery force to the neutral position is important. Without the recovery force the control device can easily be at uninteded position, and may results in unintended motion of the mobile robot. So in this study, haptic feedback force is designed to reflect the position of control device as well as the distance to obstacles. The haptic feedback force is divided to two axis and calculated as

$$
\begin{aligned}
& F_{e, x}=\left\{\begin{array}{cl}
-F_{-x} \cdot x_{h} & \text { if } x_{h}<0 \\
-F_{+x} \cdot x_{h} & \text { if } x_{h} \geq 0
\end{array}\right. \\
& F_{e, z}=\left\{\begin{array}{cl}
-F_{-z} \cdot z_{h} & \text { if } z_{h}<0 \\
0 & \text { if } z_{h} \geq 0
\end{array}\right.
\end{aligned}
$$

In order to generate the environmental haptic force in the direction away from obstacles the ultrasonic sensors of robot are divided into 3 groups (left, right and front), and $F_{-x}, F_{-y}$ and $F_{-z}$ in Eqs. 10 and 11 are calculated as

$$
\begin{aligned}
& F_{-x}=\max _{i=1}^{2}\left[F_{x i}\right] \\
& F_{+x}=\max _{i=5}^{6}\left[F_{x i}\right] \\
& F_{-z}=\max _{i=2}^{5}\left[F_{z i}\right]
\end{aligned}
$$


In previous studies linearly varying environmental feedback force as in Eq. 15 was used. In this study exponentially varying environmental force as in Eq. 16 is proposed. The reason for exponentially varying feedabck force is that human's sensitivity to gradual increase of haptic force is dull. So, in order to give a definite notion of approaching obstacle, drastic increase of haptic force is necessary.

$$
\begin{aligned}
& \left(F_{x i}, F_{z i}\right)=\left(k \cdot d_{i} \cos \theta_{i}, k \cdot d_{i} \sin \theta_{i}\right) \\
& \left(F_{x i}, F_{z i}\right)=\left(k_{1} \cdot\left(d_{i} \cos \theta_{i}\right)^{k_{2}}, k_{1} \cdot\left(d_{i} \sin \theta_{i}\right)^{k_{2}}\right) \\
& \text { where } d_{i}=\left\{\begin{array}{cc}
r_{\max }-r_{i} & r_{i}<r_{\max } \\
0 & r_{i} \geq r_{\max }
\end{array}\right.
\end{aligned}
$$

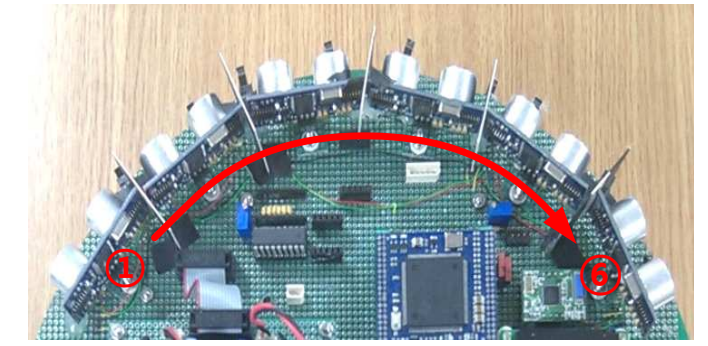

(a)

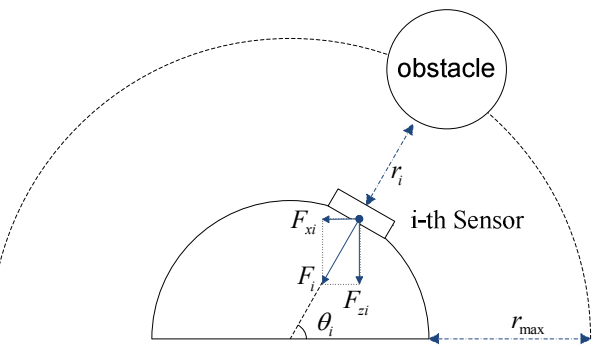

(b)

Fig. 3 Ultrasonic sensors attached to the mobile robot (a) and force generated by i-th sensor(b)

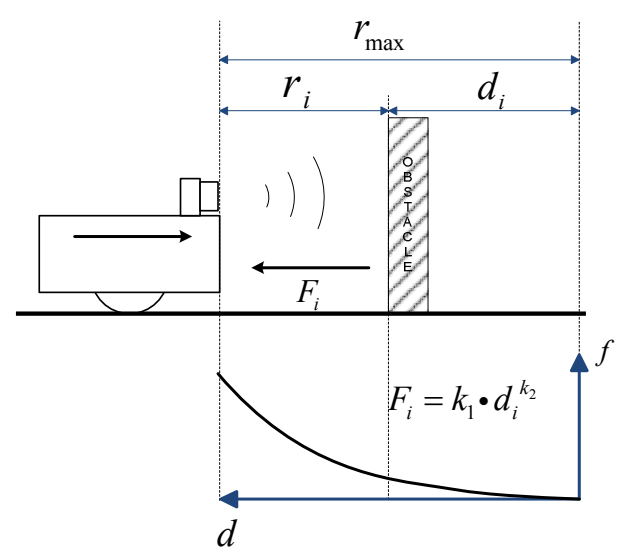

Fig. 4 Scheme for rendering feedback force based on distance to approaching obstacle.

\section{Experiments}

Experiment Design. A series of experiment for proposed haptic feedback control sheme was designed and executed. Hardware for the experiment is shown in Fig. 4.

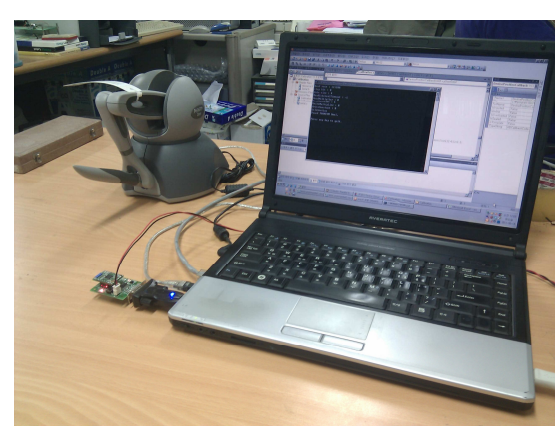

(a)

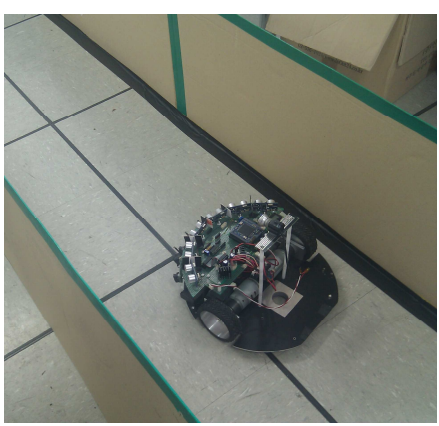

(b)

Fig. 4 Master control device that includes haptic device and computer (a), and slave system, navigating mobile robot placed in straight path (b) 
Teleoperation experiment for navigation of the mobile robot on a narrow straight path bounded by 2 paralle walls is planned. Human operator controls haptic device so that the robot can run on the center line while keeping away from the wall (obstacle). The mean squared error of robot path from the center line

$$
e=\frac{1}{T} \int_{0}^{T}\left|x_{d e s}-x_{p o s}\right|^{2} d t, \quad x_{d e s}=0
$$

is calculated to evaluate the proposed haptic feedback algorithms.

The experiments were done for 4 different conditions with exponential and linear haptic feedabck, and with and without the recovery haptic force as described in Table 1.

Table 1. Experimentla condition

\begin{tabular}{|c|c|c|}
\hline & environmental haptic force & recovery haptic force \\
\hline M1 & Exponential & no \\
\hline M2 & Linear & no \\
\hline M3 & Exponential & yes \\
\hline M4 & Linear & yes \\
\hline
\end{tabular}

Experiment Result. Mobile robot trajectory as it is placed between 2 parallel linear walls as in Fig 4 b) is recorded and the typical examples are shown in Fig. 5 for exponential environmental force feedback (M1) and linear environmental force feedback (M2) cases. It is easily recognized that the performance of exponential feedback is better than linear feedback as the error from central position is smaller.

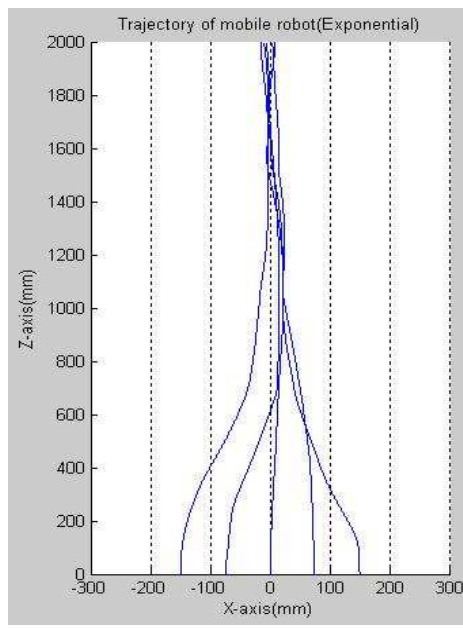

(a)

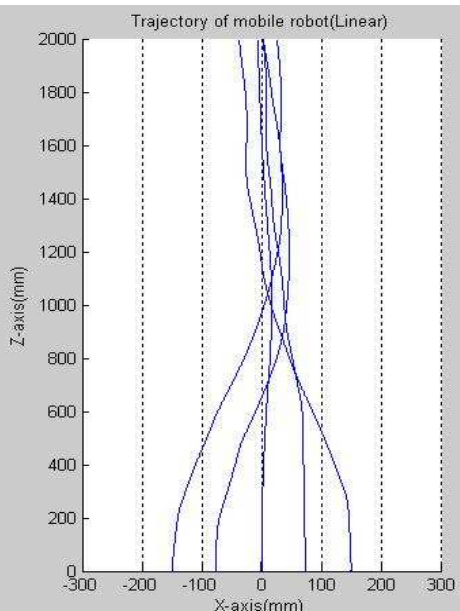

(b)

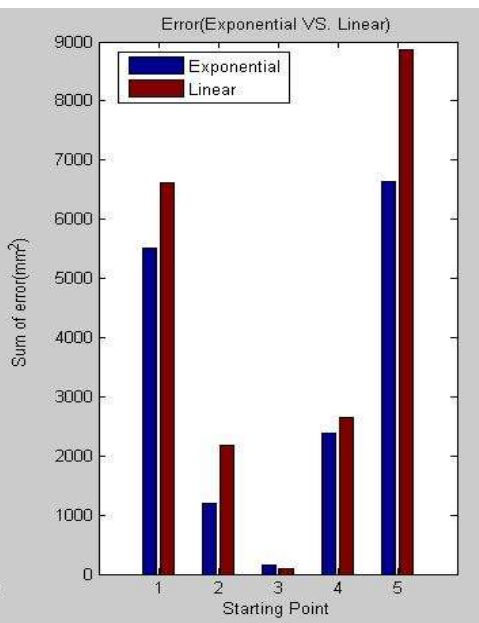

(c)

Fig. 5 Typical examples of trajectory of mobile robot with M1 method(a) and M2 method(b) and errors from the central postion for each method (c).

In Fig. 6(a) average errors for exponential feedback and linear feedback is compared. As seen in Fig. 6(a) exponential feedback is more effective than linear feedback in the majority of cases. Also, Fig. 6(b) shows that the error becomes smaller with application of recovery force. 


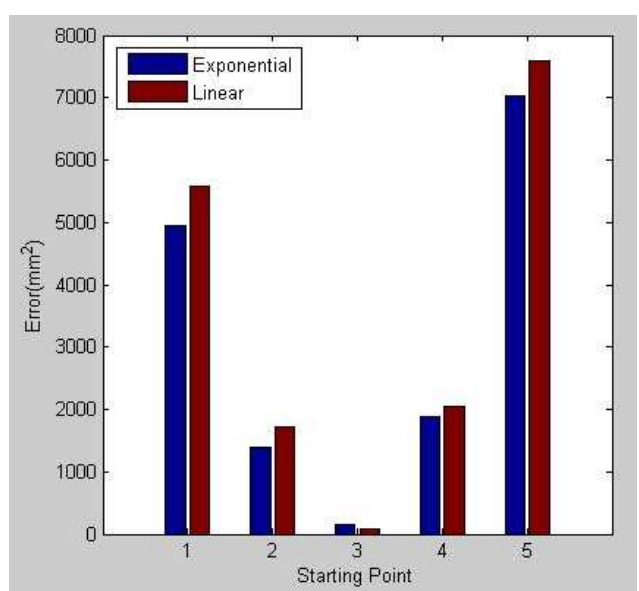

(a)

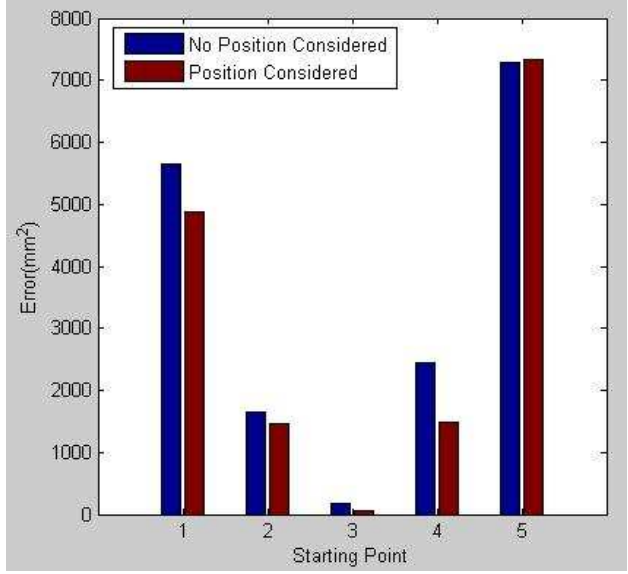

(b)

Fig. 6 Comparison of exponential and linear environmental force feedback for different starting points (a), Effect of recovery force to trajectory error (b).

\section{Conclusion}

In this study a haptic teleoperation scheme based on the exponential haptic feedback force was proposed and its effectiveness was verified through a series of experiment on teleoperation of mobile robot. The experimental result proved improved collision avoidance capability of the proposed scheme. The proposed haptic feedback control scheme is expected to provide more effective and safer operation in many teleopration applications.

\section{References}

[1] Q. Lin and C. Kuo, "Virtual tele-operation of underwater robots," in Proceedings of IEEE International Conference on Robotics and Automation, Albuquerque, NM, USA, 1997.

[2] J. P. K. Kim, H. Lee and M. Yang, "Robotic contamination cleaning system," in IEEE Conference on Intelligent Robots ans Systems, Lausanne, Switzerland, October 2002.

[3] Xinxing Tang and Hironao Yamada, "Haptic Interaction in Tele-operation Control System of Construction Robot Based on Virtual Reality," in Proceedings of the IEEE International Conference on Mechatronics and Automation, Changchun, China, 2009.

[4] Ben Horan and Saeid Nahavandi, "Intuitive Haptic Control Surface for Mobile Robot Motion Control," in Proceedings of the IEEE International Workshop on Safety, Security and Rescue Robotics, Sendai, Japan, 2008.

[5] I. Farkhatdinov, J-H. Ryu, J. Poduraev, "A user study of command strategies for mobile robot teleoperation," J. on Intelligent Service Robotics, Volume 2, Issue 2 (2009), Page 95

[6] I. Farkhatdinov, J-H. Ryu, J. An, "A Preliminary Experimental Study on Haptic Teleoperation of Mobile Robot with Variable Force Feedback Gain.” In: Proc. of IEEE Haptics Symposium 2010, Waltham, Boston, USA, 2010

[7] S. Lee, G. S. Sukhatme, G. J. Kim, C.-M. Park, "Haptic control of a mobile robot: A user study," In Proc. of IEEE/RSJ IROS 2002, Lausanne, Switzerland, October 2002.

[8] S. Lee, G. J. Kim, G. S. Sukhatme and C. M. Park, "Effects of Haptic Feedback on Telepresence and Navigational Performance," in Proc. of Intl. Conference on Artificial Telexistence, Seoul, South Korea, 2004. 